\title{
Reflets
}

Revue d'intervention sociale et communautaire

\section{La prévention en matière d'agression à caractère sexuel en Ontario français - Un témoignage personnel}

\section{Ghislaine Sirois}

Volume 18, numéro 2, automne 2012

URI : https://id.erudit.org/iderudit/1013178ar

DOI : https://doi.org/10.7202/1013178ar

Aller au sommaire du numéro

Éditeur(s)

Reflets, Revue d'intervention sociale et communautaire

ISSN

1203-4576 (imprimé)

1712-8498 (numérique)

Découvrir la revue

Citer cet article

Sirois, G. (2012). La prévention en matière d'agression à caractère sexuel en

Ontario français - Un témoignage personnel. Reflets, 18(2), 151-160.

https://doi.org/10.7202/1013178ar d'utilisation que vous pouvez consulter en ligne. 


\section{La prévention en matière d'agression à caractère sexuel en Ontario français - Un témoignage personnel}

\section{Ghislaine Sirois}

Directrice générale d'Action ontarienne contre la violence faite aux femmes, Ottawa

Action ontarienne contre la violence faite aux femmes (AOcVF) est un regroupement provincial des Centres d'aide et de lutte contre les agressions à caractère sexuel (CALACS), de maisons d'hébergement francophones et d'autres programmes offrant des services en français en Ontario en matière de violence faite aux femmes ${ }^{1}$. Les CALACS francophones ont pour mandat l'éducation et la prévention aux agressions à caractère sexuel, et ce, dans une approche féministe et communautaire. Il y a présentement neuf CALACS francophones autonomes dans la province et deux centres bilingues où sont offerts des services en français en violence, en plus de quelques programmes de counselling en matière d'agression à caractère sexuel. Ces derniers sont situés à Ottawa, à Hawkesbury et à Timmins.

En matière d'agression à caractère sexuel, des services de soutien aux femmes sont donc disponibles en français, tant sur le plan de l'intervention, de personne à personne ou en groupe, que sur celui de la prévention. Ces services ont pour la plupart été mis en place entre 1993 et 1995. Les plus récents datent de 2008. Offerts par et pour les femmes francophones, ils visaient lors de leur mise en œuvre à pallier le manque de services en français dans les institutions de langue anglaise. 
Le présent article retrace quelques faits saillants qui ont marqué mes trente-trois années de militantisme à œuvrer contre la violence faite aux femmes et contre la violence sexuelle en Ontario. On y voit le rôle que l'AOcVF, avec les CALACS et ses autres groupes membres, joue depuis plusieurs années dans l'offre de services et dans le développement de stratégies de sensibilisation à la prévention de la violence à caractère sexuel, notamment auprès des communautés de langue française en Ontario.

\section{Une offre de services en français}

En adoptant la Loi 8 sur les services en français, il y a 25 ans de cela, le gouvernement de l'Ontario reconnaissait l'apport historique des francophones et l'importance de préserver l'héritage francophone dans la province. Cette loi oblige le gouvernement à offrir des services en français dans les régions "désignées ", c'est-à-dire, des régions où au moins $10 \%$ de la population est francophone ou qui comptent plus de 5000 francophones. Un service en français de qualité comprend plusieurs éléments : l'accès à un niveau égal à celle de la majorité; l'accès à une documentation adaptée et à jour; la possibilité d'avoir accès à l'entièreté, et non à une partie, des services offerts par un organisme.

Grâce à cette loi et au soutien de partenaires communautaires qui offrent des services de prévention des agressions à caractère sexuel, des Ontariennes ont réussi à développer le réseau des CALACS francophones. Issus de cette base militante, ces derniers interviennent à la fois directement auprès des femmes non seulement en matière de soutien, mais également de sensibilisation, de prévention, d'éducation et de développement communautaire.

Pour que les CALACS puissent offrir des services à long terme, il a été proposé d'en faire des entités autonomes, autogérées par des femmes francophones. Ce principe d'autonomie permet de leur garantir une vision et une direction répondant aux besoins des femmes de la communauté. L'autonomisation est un principe fondamental de l'intervention féministe qui vise à redonner 
aux femmes le pouvoir et le contrôle de leur vie. Sur le plan collectif, elle offre également la possibilité aux organismes de mieux contrôler les ressources mises à la disposition des femmes francophones et de travailler au développement communautaire. Ainsi, les CALACS ont pu mettre en place plusieurs stratégies de prévention. Nous les présentons dans les prochains paragraphes.

\section{Un travail de prévention}

Pour comprendre le travail de l'AOcVF et des CALACS, il faut d'abord savoir ce que l'on entend par prévention et pour cela, il est nécessaire de se référer aux cadres d'analyse du Centre ontarien de prévention des agressions (Copa) (2011) et aux travaux de Haskell (2011) qui énoncent divers niveaux de prévention.

Le premier niveau porte sur la prévention des agressions à caractère sexuel et permet d'offrir aux femmes des services directs en français. Ce niveau propose de soutenir et d'informer les femmes afin de réduire leur état de vulnérabilité et, à notre avis, c'est là que se sont surtout concentrées les énergies de l'AOcVF et de ses groupes membres au cours des vingt dernières années. Ils se sont assurés d'une offre de services de qualité en français, et cela, grâce à la formation des intervenantes. Ces dernières ont désormais accès à du matériel de formation en français disponible en ligne ${ }^{2}$. Des guides d'intervention auprès des enfants témoins, des aînées, des immigrantes et des femmes vivant avec un handicap ont été élaborés dans le but de diminuer les méfaits de la violence dont ces personnes peuvent être victimes.

La sensibilisation communautaire se situe au deuxième niveau de la prévention des actes à caractère sexuel. L'objectif consiste dans cette perspective à vouloir transformer les normes sociales et les attitudes de violence à caractère sexuel. Ainsi, les CALACS ont travaillé au changement des mentalités qui voulaient que les femmes soient en quelque sorte responsables des agressions qu'elles subissaient, soit à cause de leur habillement ou de leurs activités. 
L'AOcVF a soutenu des initiatives comme celle du Centre Victoria pour Femmes à Sudbury, lequel a développé un programme visant à prévenir la victimisation des jeunes femmes. L'outil Filles fantastiques ${ }^{3}$ est désormais utilisé dans plusieurs régions par des CALACS francophones. L'AOcVF a également produit un manuel ${ }^{4}$ à l'intention des femmes de 65 ans et plus, lequel est aussi utilisé dans plusieurs CALACS et contient une série d'ateliers. Récemment, dans la région de Prescott Russell, le Centre Novas a mené une campagne de visibilité, Un mille en talons hauts ${ }^{5}$, impliquant les hommes et les femmes de la communauté.

Au troisième niveau de la prévention, l'intervention vise le changement des lois, des systèmes et des programmes qui perpétuent la violence envers les femmes. Par exemple, nous sommes intervenues lors des consultations sur la sécurité des femmes dans les milieux de travail, en particulier, à leur sécurité face au harcèlement sexuel. Nous avons rencontré les décideurs et décideuses pour faire valoir les besoins des femmes victimes de violence dans les débats sur la garde légale des enfants, où trop souvent on ne croit pas les femmes qui dénoncent la violence sexuelle perpétrée contre elles par un conjoint.

\section{Les dossiers discutés par les CALACS}

Depuis 1994, deux rencontres annuelles ont été organisées avec les intervenantes francophones au niveau provincial. À chacune de ces rencontres, les intervenantes ont discuté des enjeux d'actualité relatifs aux dossiers de la violence faite aux femmes : les nouvelles approches en intervention; la pauvreté; la prostitution; l'élimination du registre des armes à feu; et les nouvelles dispositions en matière de droit de la famille. Les échanges ont également porté sur l'amélioration de l'accès aux services en français à travers la province. Les CALACS ont travaillé étroitement avec le Mouvement ontarien des femmes immigrantes (MOFIF) sur les services à offrir aux survivantes de viols en temps de conflits armés. Celles-ci sont nombreuses à provenir des pays de la Francophonie. Des chercheuses ont aussi été invitées pour discuter de l'hypersexualisation des filles et du syndrome post-traumatique présent chez les survivantes d'agression à caractère sexuel. 


\section{Le dossier sur la prostitution}

Nous avons préparé une analyse de la prostitution ${ }^{6}$ et nous nous sommes prononcées en faveur de son abolition. Contrairement aux arguments de l'autre partie qui avance que la légalisation rendrait la prostitution plus sécuritaire, nous croyons que la prostitution est, en soi, une forme de violence envers les femmes et que cette violence constitue une violation des droits de la personne, des femmes pauvres en particulier. Notre analyse arrive à la conclusion que la prostitution n'est pas un "travail comme les autres ». Pour faire valoir cette position, nous avons adhéré à une coalition nationale qui sera prochainement entendue dans cette cause devant la Cour suprême.

\section{Le dossier sur le harcèlement sexuel en milieu de travail}

Jusqu'à ce jour, en tant qu'organismes francophones, les CALACS ont disposé de très peu de moyens pour élaborer des campagnes de sensibilisation. Au cours des années, nous avons tout de même traduit et adapté des outils de sensibilisation et de prévention : une vidéo, Changer les choses, sur le harcèlement sexuel en milieu de travail, des affiches, des sous-verres distribués dans les bars portant sur les drogues du viol. Utilisant les outils du $\mathrm{Copa}^{7}$, certains CALACS font également un important travail d'éducation et de sensibilisation au sein des communautés et des écoles.

\section{Les défis de l'information et de la prévention en situation minoritaire}

Où les femmes vont-elles chercher leurs informations? Généralement au sein de leur communauté d'appartenance. Un des défis prioritaires des CALACS porte donc sur la sensibilisation et l'accompagnement des francophones vivant en situation linguistique minoritaire en Ontario. La sensibilisation de la communauté francophone s'avère doublement difficile, en raison d'une part de l'isolement inhérent à la violence et, d'autre 
part, de sa situation de minoritaire. Pour cela, les CALACS doivent disposer non seulement d'outils de sensibilisation efficaces, mais d'un accès à des agences de diffusion (journaux, quotidiens et médias francophones électroniques) reconnues par la communauté. Pour les CALACS qui ont peu de moyens de diffusion, faire connaitre leurs messages constitue un grand défi dans le développement de campagnes de sensibilisation. Aussi, le sentiment d'appartenance peut parfois jouer un rôle important lors des campagnes de sensibilisation.

Le sentiment d'appartenance apparaît plus significativement lors de la médiatisation de situations tragiques relatives à la violence. Des francophones paraitront moins touchés face à une telle agression survenue dans le groupe majoritaire et nieraient presque que cela puisse se produire dans "leur » communauté, comme si cela n'arrivait qu'ailleurs... Il est donc essentiel de pouvoir s'assurer que les canaux que nous empruntons soient accessibles aux francophones.

\section{Prévention et sensibilisation}

Essentiellement, ce qui ressort sur le plan de la prévention et de la sensibilisation communautaire, c'est qu'il y a beaucoup de travail à accomplir sur le terrain : une femme à la fois, une classe et une école à la fois, un groupe communautaire ou un milieu de travail à la fois... La sensibilisation sociale de masse en français risque de ne pas fonctionner si les moyens de diffusion, si les messages ne tiennent pas compte de la spécificité des communautés de langue française. Il ne faut pas qu'on puisse prétendre que "ce n'est pas nous, que c'est une affaire pour les autres ", il faut que ça parle aux communautés, que ça les rejoigne pour qu'elles comprennent que la violence peut survenir n'importe où. Nous avons du travail à faire sur le terrain, un peu comme on le fait pour la campagne Voisin-es, ami-es et familles ${ }^{4}$ où on cherche à rejoindre les personnes des communautés de langue française, un groupe à la fois.

La question de l'identité francophone est pour nous une question fondamentale. Nous avons beaucoup travaillé à développer 
des approches qui incitent le gouvernement de l'Ontario à remplir ses obligations d'offrir des services en français de qualité égale. À cet égard, nous sommes heureuses de constater que le Plan d'action de l'Ontario contre les agressions sexuelles indique dans ses politiques la mise sur pied d'un comité mixte (ministères et communauté) pour mieux satisfaire les besoins particuliers des femmes francophones.

\section{Les stratégies de sensibilisation et de protection des femmes}

Nous faisons le pari que plus nos services et programmes seront reconnus, plus nous recevrons d'appels de femmes demandant des services. Serons-nous en mesure de leur offrir les services dont elles ont besoin avec les ressources en place? Qu'advient-il des femmes qui ont besoin de services à plus long terme - car nos interventions sont le plus souvent de courte durée - et qui ne vivent pas, par exemple, à Ottawa, à Hawkesbury ou à Timmins? Où pouvons-nous les diriger pour obtenir des services en français à plus long terme?

Que faire des jeunes filles qui ne seraient pas rejointes par nos efforts de sensibilisation? À qui iront-elles parler? Avec l'obligation de faire un rapport à la Société d'aide à l'enfance, nous nous demandons si cette politique ne ralentit pas les dénonciations des méfaits auprès des jeunes qui peuvent en arriver à craindre des représailles. Mais aussi, est-ce que les travailleuses sociales qui œuvrent dans les écoles se sentent bien outillées pour soutenir ces jeunes?

Nous nous interrogeons également sur les effets possibles de la nouvelle Loi C-22 portant sur la protection des enfants contre l'exploitation sexuelle à savoir si cette loi ne vise pas plutôt le " contrôle » de la sexualité des jeunes à des fins morales. À tous ces questionnements, il n'y a encore que trop peu de réponses.Aussi, le 19 mai 2011, les intervenantes des CALACS et d'autres services de prévention de la violence à caractère sexuel, ainsi que des groupes 
communautaires pour les femmes handicapées, immigrantes, âgées et jeunes, comme le Copa, le Mouvement ontarien des femmes immigrantes, le Phénix/Opale ou la Fédération de la jeunesse franco-ontarienne, se sont rencontrées pour entamer une réflexion en profondeur sur le travail d'éducation publique à faire en matière de violence à caractère sexuel.

Certains éléments qui sont ressortis des discussions lors de cette journée ont porté sur le choix des messages que nous voulons livrer à notre communauté. Nous avons beaucoup parlé des voies à prendre pour bien faire passer notre message lors de nos campagnes de sensibilisation. Le groupe est d'avis que nos messages et les images choisies doivent être positifs, montrer des femmes « fortes ", bien dans leur peau, plutôt qu'une victime qui a des marques de blessures et encore moins les stéréotypes de femmes ivres étendues sur un divan. Nous devons montrer des images de femmes qui reflètent nos valeurs. Les images peuvent être sexy, comme dans "Yes makes sex hot", mais elles ne doivent pas être sexistes, ni illustrer la soumission ou des corps de femmes dénudés, etc.

Nous avons aussi parlé d'utiliser des personnes influentes qui seraient des alliées dans la transmission de nos messages. Cette idée a été rejetée en faveur de messages et d'images qui indiquent que la prévention de la violence à caractère sexuel c'est l'affaire de tous et de toutes. Nous avons tous et toutes le pouvoir de travailler à changer les choses. Les intervenantes ont aussi indiqué avoir besoin de formation et d'outils pour faire le travail de terrain dans la communauté de langue française. C'est ainsi que nous avons mis en œuvre la campagne Voisin-es, ami-es et familles $^{8}$ qui propose des outils de formation destinés aux agentes responsables de la campagne.

\section{Pour conclure}

Pour parvenir à nos objectifs de sensibilisation, de prévention et d'éducation, il est nécessaire de travailler en concertation. Nous avons à rejoindre la communauté de langue française dans toute 
sa diversité afin d'établir une stratégie efficace et constructive. Nous avons besoin d'effectuer des changements en profondeur, ce qui va devoir se faire sur une longue période de temps et dans la continuité. Il faudra consacrer les énergies requises, en particulier dans des communautés où les médias sont plutôt absents, et réaliser un travail de sensibilisation de longue haleine. Finalement, il reste énormément à accomplir, et de façon plus systémique en ce qui concerne la prostitution, la pornographie et l'hypersexualisation.

Voici donc de mémoire ces quelques faits saillants qui ont marqué mes trente-trois années de militantisme à œuvrer contre la violence faite aux femmes et contre la violence sexuelle. Je me console en pensant qu'à l'époque, nous nous disions : «il faut parler aux policiers, aux juges... », « il faut changer la loi définissant le viol de façon trop étroite "... Nos groupes souffraient du manque d'outils d'intervention et de sensibilisation. Seuls quelques ouvrages de féministes américaines ou françaises avançaient de grands principes, mais malheureusement peu applicables dans la réalité de tous les jours. Une fois par année, nous intervenions bien timidement dans les classes. Bref, nous avons fait un bon bout de chemin depuis ce temps où tout était à faire. Ce qui est certain, cependant, c'est que nous n'avons malheureusement pas encore fini le travail!

\section{Notes}

1 Pour une liste complète des services, consulter le site d'Action ontarienne contre la violence faite aux femmes, www.aocvf.ca, sous la rubrique Services aux femmes.

2 Ce matériel portant sur la violence conjugale et la violence à caractère sexuel et a été élaboré en partenariat avec une professeure de l'École de service social de l'Université Laurentienne et le Consortium national de formation en santé. Consulter le site www.ressources-violence.org

3 Pour en connaitre davantage sur les ateliers de Filles fantastiques, consulter le site de la Fondation Filles d'Action à l'adresse http://girlsactionfoundation.ca/fr/guide-des-ateliers-de-fillesfantastiques-une-approche-artistique-\%C3\%A0-la-programmation-pour-les-jeunes-femmes.

4 La violence faite aux femmes aînées francophones: Une problématique à cerner, des services en français à offrir, 2009, $61 \mathrm{p}$.

5 Consulter le site de la campagne à l'adresse http://www.centrenovas.ca/pdf/marche_hommes_2012.pdf 
6 DAY, Shelagh (2008). La prostitution, une violation des droits humains des femmes pauvres, Action ontarienne contre la violence faite aux femmes, (traduction Diane Archambault], Ottawa, réf. du $1^{\text {er }}$ août 2012, http://aocvf.ca/documents/Prostitution_v._fran_FINALE.pdf

7 Consulter le site du COPA à l'adresse www.infocopa.com

8 Consulter le site de la campagne Voisin-es, ami-es et familles à l'adresse www.voisinsamisetfamilles.ca

\section{Bibliographie}

CENTRE ONTARIEN DE PRÉVENTION DES AGRESSIONS (2011). Rapport final : Projet sur la sensibilisation en matière de violence faite aux femmes, marrainé par Action ontarienne contre la violence faite aux femmes, Toronto, Centre ontarien de prévention des agressions, $63 \mathrm{p}$.

HASKELL, Lori (2011). Les principales pratiques exemplaires des campagnes efficaces de prévention de la violence à caractère sexuel, Sommaire, réf. du $1^{\mathrm{er}}$ août 2012, http://www.forumviolenceacaracteresexuel.ca/ 